\title{
Do all Constructive Strongly Monotone Intertemporal Orders exhibit Impatience?
}

\author{
Kuntal Banerjee, Ram Sewak Dubey ${ }^{\dagger}$
}

September 2012

\begin{abstract}
In this paper we show that if a strongly monotone intertemporal order exhibits no preference towards the advancement of timing of future utility on any infinite utility (payoff or consumption) stream, then (i) the existence of such an order must involve non-constructive ways and (ii) the order cannot be represented by a real-valued function.
\end{abstract}

JeL Codes: D90

Keywords: Impatience, Axiom of Choice, Non-Ramsey Set

*Corresponding Author: Barry Kaye School of Business, Room KH 105, Florida Atlantic University, Boca Raton FL 33431, USA. Email: kbanerje@fau.edu or kuntal.banerjee@gmail.com

${ }^{\dagger}$ Department of Economics and Finance, Montclair State University, Montclair, NJ, 07042; Email: dubeyr@mail.montclair.edu. 


\section{Introduction}

In this paper we formalize the notion that the task of explicitly describing (constructing) a strongly monotone (a formal definition is provided in section 2.2) order on the space of infinite sequences of $\{0,1\}$ (interpreted as sequence of utilities corresponding to "good" and "bad" states) implies the existence of streams at which the advancement of timing of future satisfaction is favored (impatience) over the status quo (this concept is formalized in section 2.3). Informally, if sensitivity towards generational payoff is demanded of an evaluation of infinite streams, then there is no escaping the incidence of impatience, unless one uses non-constructive mathematical tools like the Axiom of Choice. We use techniques from Lauwers $(2010,2012)$ to formalize the idea that if a strongly monotone order does exhibit pure patience at every point in the program space, then such an order is necessarily non-constructive.

To wit, a non-constructive proof typically deduces the existence of a mathematical object without providing means of specifying it. In Svensson (1980), it was shown, using the Axiom of Choice, that a complete ordering of infinite utility streams satisfying strong monotonicity and anonymity (the property of being indifferent to finite permutation of generational utilities) is possible. If an order satisfies the anonymity condition it implies that the defined order does not exhibit impatience at any infinite utility stream. However, there seems to be no known way of escaping the use of the Axiom of Choice in Svensson's order. In Banerjee and Mitra (2007) it was demonstrated that every strongly monotone order that is representable (by a real-valued function) must exhibit some impatience. These two results lead us to the following question: Does every constructive or representable intertemporal order on infinite utility streams satisfying strong monotonicity exhibit some impatience?

We use the techniques introduced to economics by Lauwers $(2010,2012)$ to answer this question; in particular, we show that the existence of an intertemporal order satisfying strong monotonicity that does not exhibit impatience at any infinite utility stream entails the existence of a non-constructive object, a Non-Ramsey set. Section 2 presents preliminaries, section 3 states and proves the main result and section 4 illustrates a crucial example.

\section{Preliminaries}

\subsection{Order Theoretic Definitions}

Let $\mathbb{N}$ denote the set of natural numbers. The set of infinite utility streams is the set of all sequences with at least two elements; we let $X=Y^{\mathbb{N}}$ with $Y \subset \mathbb{R}$, a non-empty subset of the real line. In this note the only restriction on $Y$ would be that it contain at least two distinct elements, say 0 and 1 . In view of this, we will work with $Y=\{0,1\}$ and for this choice of $Y$, infinite utility streams would then be the set of sequences with each term of the sequence being either 0 or 1 . While we refer to the sequence space $X$ to contain utility streams, the framework is general enough to accommodate 
other scenarios as well, for example outcomes indexed by periods in a dynamic game, returns from investment decision, consumption in a dynamic economic model among many others.

Given infinite utility streams $\mathbf{x}, \mathbf{y}$ in $X$ we write $\mathbf{x} \geq \mathbf{y}$ if $x_{n} \geq y_{n}$ for all $n \in \mathbb{N}$ and denote by $\mathbf{x}>\mathbf{y}$ if $\mathbf{x} \geq \mathbf{y}$ and $\mathbf{x} \neq \mathbf{y}$.

An intertemporal order (interchangeably called a preference order on $X$ ) is a binary relation $\succsim$ on $X$ which is complete (if for any $\mathbf{x}, \mathbf{y} \in X$ either $\mathbf{x} \succsim \mathbf{y}$ or $\mathbf{y} \succsim \mathbf{x}$ holds) and transitive. Given a preference order $\succsim$ on $X$, we indicate it's asymmetric and symmetric parts by $\succ$ and $\sim$. Recall, for $\mathbf{x}, \mathbf{y} \in X$, $\mathbf{x} \succ \mathbf{y}$ implies $\mathbf{x} \succsim \mathbf{y}$ and not $\mathbf{y} \succsim \mathbf{x}$, and the symmetric relation $\mathbf{x} \sim \mathbf{y}$ is defined as $\mathbf{x} \succsim \mathbf{y}$ and $\mathbf{y} \succsim \mathbf{x}$.

An intertemporal order is representable if there is some $U: X \rightarrow \mathbb{R}$ such that for any $\mathbf{x}, \mathbf{y} \in X$, we have $\mathbf{x} \succsim \mathbf{y}$ iff $U(\mathbf{x}) \geq U(\mathbf{y})$.

\subsection{Sensitivity Condition}

The fundamental behavioral restriction we impose on intertemporal preferences is that of sensitivity to generational utilities. Let $\succsim$ be a preference order on $X$, it is said to satisfy,

Strong Monotonicity: if $\mathbf{x}, \mathbf{y} \in X$ and $\mathbf{x}>\mathbf{y}$, then $\mathbf{x} \succ \mathbf{y}$.

In accordance with the grammatical construct of the sentence we will use the phrases strongly monotonic and strongly monotone to describe orders satisfying strong monotonicity. The strong monotonicity condition is the strong Pareto condition, S2 in Diamond (1965, p.173) and Basu and Mitra (2003).

\subsection{The Impatience and Patience Condition}

We provide here a precise definition of what we mean for a preference order on $X$ to exhibit impatience at some $\mathbf{x} \in X$. Some auxiliary definitions are needed to formalize our impatience condition. Given $\mathbf{x} \in X$, and $M, N \in \mathbb{N}$, we denote by $\mathbf{x}(M, N)$ the sequence $\mathbf{x}^{\prime} \in X$ defined by,

$$
x_{M}^{\prime}=x_{N}, x_{N}^{\prime}=x_{M} \text { and } x_{n}^{\prime}=x_{n}, \forall n \neq N, M \text {. }
$$

An intertemporal order $\succsim$ is said to exhibit impatience at $\mathbf{x} \in X$, if there exist $M, N \in \mathbb{N}$ with $M>N$ such that, either

$$
\text { (i) } x_{M}>x_{N} \text { and } \mathbf{x}(M, N) \succ \mathbf{x} \text {; or (ii) } x_{M}<x_{N} \text { and } \mathbf{x} \succ \mathbf{x}(M, N) \text {. }
$$

Observe that the definition of a new sequence $\mathbf{x}(M, N)$ in (1) from some $\mathbf{x} \in X$ involves swapping oneperiod utilities corresponding to periods $M$ and $N$, ceteris paribus. The impatience condition captures the intuition that the preference order $\succsim$ exhibits a preference towards "immediate gratification". 
An intertemporal order $\succsim$ is said to exhibits no impatience on $X$ if for each $\mathbf{x} \in X$ and any $M, N \in \mathbb{N}$ with $M>N$, whenever $x_{M}>x_{N}$ we have $\mathbf{x}(M, N) \precsim \mathbf{x}$, and $x_{M}<x_{N}$ implies $\mathbf{x}(M, N) \succsim \mathbf{x}$. The terms "exhibits no impatience" and "exhibits pure patience" are used interchangeably.

In the intergenerational equity literature the demand of "equal treatment" is made on intertemporal orders. The notion of equal treatment is captured through the condition of anonymity.

An intertemporal order $\succsim$ is said to satisfy anonymity if for each $\mathbf{x} \in X$ and any $M, N \in \mathbb{N}$ we have $\mathbf{x}(M, N) \sim \mathbf{x}$. Equal treatment as captured through anonymity implies pure patience, however the two concepts are distinct. An example is provided in section 4 to illustrate this point.

\subsection{Non-Ramsey Sets}

We will now define a Non-Ramsey set and formalize what is meant by a constructive mathematical object. Let $I$ be an infinite set and for any positive integer $n$ indicate by $[I]^{n}$ the collection of all subsets of $I$ with exactly $n$ elements. Ramsey (1928) showed that for each subset $S$ of $[I]^{n}$, there exists some infinite subset $J \subset I$ such that either $[J]^{n} \subset S$ or $[J]^{n} \cap S=\emptyset$. However, Ramsey's Theorem fails if we replace $n$ with countable infinity. Formally, there is a subset $R$ of $[I]^{\infty}$ (where $[I]^{\infty}$ is the collection of subsets of $I$ each of which are of infinite cardinality) such that for each infinite subset $J \subset I$ the class $[J]^{\infty}$ intersects with $R$ as well as with $[I]^{\infty} \backslash R$. Such a set $R$ is called a Non-Ramsey set.

We will call a mathematical object constructive if its existence can be proved using the ZermeloFrankel (ZF) axioms of set theory alone (in particular, without the use of the Axiom of Choice). If the use of the Axiom of Choice (AC) is necessary to the existence of some mathematical entity, then we say that the proof of its existence is non-constructive. Mathias (1977) has shown that the existence of a Non-Ramsey set does not follow from ZF (without AC).

\section{The Main Result}

We introduce some notation needed to present the main result of the paper.

A sequence in $\mathbb{N}$, denoted $\left\langle n_{k}\right\rangle$ satisfies $n_{k}<n_{k+1}$ for $k \in \mathbb{N}$. For $n<n^{\prime}$, denote the set $\left\{n, \ldots, n^{\prime}-1\right\}$ by $\left[n, n^{\prime}[\right.$. Given a subset infinite $J \subset \mathbb{N}$, we can rewrite the elements of $J$ in increasing order, that is $J=\left\{j_{1}, j_{2}, \ldots\right\}$ with $j_{k}<j_{k+1}$ for each $k$. Hence any infinite subset of $\mathbb{N}$ can be associated with a sequence in $\mathbb{N}$. So each element in the collection of subsets of $\mathbb{N}$ with infinite cardinality (in our notation $[\mathbb{N}]^{\infty}$ ) can be viewed as a sequence. We define two functions $\mathbf{x}:[\mathbb{N}]^{\infty} \rightarrow X$ and $\mathbf{y}$ : $[\mathbb{N}]^{\infty} \rightarrow X$ as follows. For any $J=\left\{j_{1}, j_{2}, \ldots\right\} \in[\mathbb{N}]^{\infty}$ with $j_{k}<j_{k+1}$ for each $k$, we write the image $\mathbf{x}(J)=\left(x_{1}, x_{2}, \ldots\right)$ and $\mathbf{y}(J)=\left(y_{1}, y_{2}, \ldots\right)$ where,

$$
x_{n}=\left\{\begin{array}{cc}
0 & \text { for } n<j_{1} \\
0 & \text { for } n \in\left[j_{k}, j_{k+1}[\text { for } k \text { odd }\right. \\
1 & \text { otherwise }
\end{array}\right.
$$


and

$$
y_{n}=\left\{\begin{array}{cc}
0 & \text { for } n<j_{1} \\
1 & \text { for } n \in\left[j_{k}, j_{k+1}[\text { for } k \text { odd }\right. \\
0 & \text { otherwise }
\end{array}\right.
$$

Proposition 1 If there exists an intertemporal order $\succsim$ satisfying strong monotonicity that exhibits no impatience on $X$, then the set $S=\left\{N \in[\mathbb{N}]^{\infty}: x(N) \succ y(N)\right\}$ is a Non-Ramsey set.

Proof: To show that the subset $S$ of $[\mathbb{N}]^{\infty}$ is a Non-Ramsey set we consider an arbitrary infinite subset $K$ of $\mathbb{N}$. Take a infinite subset $J$ of $K$ such that $j_{2}-j_{1}<j_{3}-j_{2}$ (such a choice of subset will always exist from an infinite inductive set). Since $\succsim$ is an order on $X$, either (a) $\mathbf{x}(J) \succ \mathbf{y}(J)$ (b) $\mathbf{y}(J) \succ \mathbf{x}(J)$ or (c) $\mathbf{x}(J) \sim \mathbf{y}(J)$ must hold. In each case, namely, (a), (b) and (c) we need to show that $[K]^{\infty}$ intersects with $S$ as well as with $[\mathbb{N}]^{\infty} \backslash S$.

Proof. Step $\mathbf{1}\left[\right.$ If $\mathbf{x}(J) \succ \mathbf{y}(J)$, then there is an infinite subset $J^{\prime} \subset J$ such that $\left.\mathbf{y}\left(J^{\prime}\right) \succ \mathbf{x}\left(J^{\prime}\right)\right]$ : Consider the sequences:

$\begin{array}{ccccccc} & {\left[1, j_{1}[\right.} & {\left[j_{1}, j_{2}[\right.} & {\left[j_{2}, j_{3}[\right.} & {\left[j_{3}, j_{4}[\right.} & {\left[j_{4}, j_{5}[\right.} & \\ \mathbf{x}(J) & 0 & 0 & 1 & 0 & 1 & \ldots \\ \mathbf{y}(J) & 0 & 1 & 0 & 1 & 0 & \ldots \\ \mathbf{x}\left(J \backslash\left\{j_{2}\right\}\right) & 0 & 0 & 0 & 1 & 0 & \ldots \\ \mathbf{y}\left(J \backslash\left\{j_{2}\right\}\right) & 0 & 1 & 1 & 0 & 1 & \ldots\end{array}$

In the matrix the sequences depict finite snapshots (up to $j_{5}-1$ terms) of the the functions $\mathbf{x}$ and $\mathbf{y}$ for the infinite subset of $J$ and $J \backslash\left\{j_{2}\right\}$. We have dropped the second term in the sequence $J$ to obtain the last two rows. Using strong monotonicity, we have $\mathbf{y}(J) \succ \mathbf{x}\left(J \backslash\left\{j_{2}\right\}\right)$ and $\mathbf{y}\left(J \backslash\left\{j_{2}\right\}\right) \succ \mathbf{x}(J)$ [in each comparison the values taken beyond $j_{3}$ are identical across the compared sequences]. Together with $\mathbf{x}(J) \succ \mathbf{y}(J)$ and transitivity of $\succsim, \mathbf{y}(J) \succ \mathbf{x}\left(J \backslash\left\{j_{2}\right\}\right)$ and $\mathbf{y}\left(J \backslash\left\{j_{2}\right\}\right) \succ \mathbf{x}(J)$ yields $\mathbf{y}\left(J \backslash\left\{j_{2}\right\}\right) \succ \mathbf{x}\left(J \backslash\left\{j_{2}\right\}\right)$, as we set out to show in this step.

Step $\mathbf{2}\left[\right.$ If $\mathbf{y}(J) \succsim \mathbf{x}(J)$, then there is an infinite subset $J^{\prime \prime} \subset J$ such that $\left.\mathbf{x}\left(J^{\prime \prime}\right) \succ \mathbf{y}\left(J^{\prime \prime}\right)\right]$ : Consider the following sequences:

$\begin{array}{ccccccc} & {\left[1, j_{1}[\right.} & {\left[j_{1}, j_{2}[\right.} & {\left[j_{2}, j_{3}[\right.} & {\left[j_{3}, j_{4}[\right.} & {\left[j_{4}, j_{5}[\right.} & \\ \mathbf{x}(J) & 0 & 0 & 1 & 0 & 1 & \ldots \\ \mathbf{y}(J) & 0 & 1 & 0 & 1 & 0 & \ldots \\ \mathbf{x}\left(J \backslash\left\{j_{3}\right\}\right) & 0 & 0 & 1 & 1 & 0 & \ldots \\ \mathbf{y}\left(J \backslash\left\{j_{3}\right\}\right) & 0 & 1 & 0 & 0 & 1 & \ldots\end{array}$

We have dropped the third term in the sequence $J$ to obtain the last two rows. Note that the sequences $\mathbf{x}(J)$ and $\mathbf{y}\left(J \backslash\left\{j_{3}\right\}\right)$ are identical beyond $j_{3}$ and similarly, $\mathbf{y}(J)$ and $\mathbf{x}\left(J \backslash\left\{j_{3}\right\}\right)$ are identical beyond $j_{3}$. Let us compare $\mathbf{x}(J)$ and $\mathbf{y}\left(J \backslash\left\{j_{3}\right\}\right)$. They cannot be directly compared using strong monotonicity. However, observe that we can obtain $\mathbf{y}^{\prime}$ from $\mathbf{y}\left(J \backslash\left\{j_{3}\right\}\right)$ by swapping the 1 's from $\left[j_{1}, j_{2}\left[\right.\right.$ with the 0 's in the range $\left[j_{2}, j_{3}\left[\right.\right.$. Since $\succsim$ exhibits no impatience, we must have $\mathbf{y}^{\prime} \succsim \mathbf{y}\left(J \backslash\left\{j_{3}\right\}\right)$. Now compare $\mathbf{y}^{\prime}$ to $\mathbf{x}(J)$ and note that the profiles are identical in all periods except for some indices 
in the range $\left[j_{2}, j_{3}\left[\right.\right.$, for which $\mathbf{y}^{\prime}$ values are 0 and $\mathbf{x}(J)$ values are 1 [this is a direct consequence of $j_{2}-j_{1}<j_{3}-j_{2}$, since it guarantees that in the swaps described in the last line, some 0's remained in the range $\left[j_{2}, j_{3}\left[\right.\right.$ in obtaining $\mathbf{y}^{\prime}$ from $\left.\mathbf{y}\left(J \backslash\left\{j_{3}\right\}\right)\right]$. So by strong monotonicity, it must be true that $\mathbf{x}(J) \succ \mathbf{y}^{\prime}$ and by transitivity of $\succsim$ along with $\mathbf{y}^{\prime} \succsim \mathbf{y}\left(J \backslash\left\{j_{3}\right\}\right)$ we conclude $\mathbf{x}(J) \succ \mathbf{y}\left(J \backslash\left\{j_{3}\right\}\right)$. A symmetric argument shows that $\mathbf{x}\left(J \backslash\left\{j_{3}\right\}\right) \succ \mathbf{y}(J)$. Now $\mathbf{y}(J) \succsim \mathbf{x}(J)$ implies (using transitivity of $\succsim) \mathbf{x}\left(J \backslash\left\{j_{3}\right\}\right) \succ \mathbf{y}\left(J \backslash\left\{j_{3}\right\}\right)$, concluding the analysis of this step.

In case (a), using step 1 , we get $\left(J \backslash\left\{j_{2}\right\}\right) \in[K]^{\infty} \cap\left([\mathbb{N}]^{\infty} \backslash S\right)$, whereas $J \in[K]^{\infty} \cap S$. For cases (b) and (c), if $\mathbf{y}(J) \succ \mathbf{x}(J)$ or $\mathbf{y}(J) \sim \mathbf{x}(J)$, then $J \in[K]^{\infty} \cap\left([\mathbb{N}]^{\infty} \backslash S\right)$ and using the analysis in step 2 (since $\mathbf{y}(J) \succsim \mathbf{x}(J)$ holds in both cases) we have $\left(J \backslash\left\{j_{3}\right\}\right) \in[K]^{\infty} \cap S$ as was needed to demonstrate that $S$ is a Non-Ramsey set.

Before stating the theorem, we state Theorem 1 from Banerjee and Mitra (2007) for reference.

Theorem 1 (Banerjee and Mitra (2007)) Let $\succsim$ be a representable, intertemporal order satisfying strong monotonicity, then there are a continuum of points in $X$ at which $\succsim$ exhibits impatience.

We are now ready to state the main theorem, the proof of which follows easily from Proposition 1 and Theorem 1 and is not explicitly stated.

Theorem 2 Let $\succsim$ be an intertemporal order satisfying strong monotonicity. If (i) the existence of $\succsim$ is guaranteed through constructive methods, or $(b) \succsim$ is representable, then $\succsim$ must exhibit impatience at some $x \in X$.

\section{Remarks:}

(i) Method of Proof : The proof technique used in Proposition 1 was introduced to economics by Lauwers (2010). We have used his innovation to obtain a very stark result on the nature of impatience and the role of the use of non-constructive methods to define evaluations of infinite payoff streams. In Lauwers (2010) it was shown that orders satisfying anonymity and strong monotonicity are necessarily non-constructive. Our requirement of exhibiting pure patience is not identical to the anonymity condition. There are strongly monotone orders that exhibit pure patience at every utility stream but are not anonymous, an example of such an order is provided in section 4 . In particular, the example also demonstrates that the conclusion in Theorem 2 above cannot be directly inferred from Lauwers (2010).

(ii) Generalization of Theorem 2: In the statement of Proposition 1 and Theorem 2 we make use of strong monotonicity. Our results also hold for a weaker sensitivity requirement, namely infinite Pareto. This condition can be stated as follows: if $\mathbf{x}, \mathbf{y} \in X$ and $x_{n} \geq y_{n}$ for all $n \in \mathbb{N}$ and for some subsequence $\left\{N_{k}\right\}$ of $\mathbb{N}$ the inequality is strict, then $\mathbf{x} \succ \mathbf{y}$. The method of proof presented in Proposition 1 readily generalizes to incorporate the weaker sensitivity requirement. We have deliberately chosen the stronger condition as it simplifies the exposition significantly in our view without losing the power of the tool that we employ. For the argument to go through with the weaker Pareto condition one needs to take away from the original sequence ( $J$ in the proof) an infinite subsequence in steps 1 and 2, the details are available with the authors upon request. 


\section{Example}

In this section we present the example of a preference order on $X$ which (a) exhibits no impatience at any $\mathbf{x} \in X$ (b) does not satisfy anonymity and (c) is strongly monotone. Based on our analysis so far (Proposition 1 in particular), we are assured that an explicit description (one that is devoid of the use of Axiom of Choice or such contrivances) of such an order will not be possible.

We begin with some additional notation and a well known result from order theory. A binary relation $\succsim$ on $X$ satisfying transitivity and reflexivity (for any $\mathbf{x} \in X$ if $\mathbf{x} \sim \mathbf{x}$ holds) is called a preorder. As before for any binary relation the asymmetric and symmetric parts of $\succsim$ are denoted $\succ$ and $\sim$ respectively. Given two binary relations $\succsim$ and $\gtrsim$, we say that $\succsim$ is a subrelation to $\gtrsim$ (with asymmetric and symmetric components $\gg$ and $\approx$ ) if whenever $x \succ y$ we have $x \gg y$, and if $x \sim y$, then $x \approx y$ holds. Szpilrajn's Lemma (Szpilrajn (1930)) famously states that a preorder $\succsim$ on a set can be extended to a complete order $\gtrsim$ on $X$, such that $\succsim$ is a subrelation to $\gtrsim$.

Define

$$
\mathbf{x} \succ \mathbf{y} \text { iff there is } N \text { such that } \sum_{k=1}^{n} k\left(x_{k}-y_{k}\right)>0 \text { for all } n \geq N
$$

and

$$
\mathbf{x} \sim \mathbf{y} \text { iff there is } N \text { such that } \sum_{k=1}^{n} k\left(x_{k}-y_{k}\right)=0 \text { for all } n \geq N,
$$

and let $\mathbf{x} \succsim \mathbf{y}$ iff $\mathbf{x} \succ \mathbf{y}$ or $\mathbf{x} \sim \mathbf{y}$. It is easy to check that $\succsim$ is a preorder on $X$. Reflexivity is obvious. We can also check that the symmetric and asymmetric parts in (5) and (6). The explicit verification of the transitivity of $\succ$ established next. The transitivity of $\succsim$ follows using a very similar method.

To demonstrate transitivity of $\succ$, suppose $\mathbf{x} \succ \mathbf{y}$ and $\mathbf{y} \succ \mathbf{z}$ for some $\mathbf{x}, \mathbf{y}, \mathbf{z} \in X$. We need to show

that $\mathbf{x} \succ \mathbf{z}$. There is some $N_{1}, N_{2}$ such that $\sum_{k=1}^{n} \mathrm{k}\left(\mathrm{x}_{k}-y_{k}\right)>0$ for all $n \geq N_{1}$ and $\sum_{k=1}^{n} k\left(y_{k}-z_{k}\right)>0$ for all $n \geq N_{2}$. Denote $\max \left\{N_{1}, N_{2}\right\}$ by $N$ and for any $n \geq N$ we have

$$
\begin{aligned}
\sum_{k=1}^{n} k\left(x_{k}-z_{k}\right) & =\sum_{k=1}^{n} k\left(x_{k}-y_{k}+y_{k}-z_{k}\right) \\
& =\sum_{k=1}^{n} k\left(x_{k}-y_{k}\right)+\sum_{k=1}^{n} k\left(y_{k}-z_{k}\right)>0
\end{aligned}
$$

so $\mathbf{x} \succ \mathbf{z}$ by (5). It is easy to see that a similar analysis guarantees that $\succsim$ is transitive. Hence, $\succsim$ is a preorder on $X$. By Szpilrajn's Lemma there is complete order $\gtrsim$ (with asymmetric and symmetric components $\gg$ and $\approx$ ) on $X$ such that $\succsim$ is a subrelation to $\gtrsim$. We will show that $\gtrsim$ does not exhibit impatience at any $\mathbf{x} \in X$, it is not anonymous and it satisfies strong monotonicity.

To show that $\gtrsim$ does not exhibit any impatience and does not satisfy anonymity, take $\mathbf{x}$ be a nonconstant sequence. So there is some $M>N$ such that $x_{M} \neq x_{N}$. Since $\succsim$ is a subrelation to $\gtrsim$ it is sufficient to show that whenever $x_{N}>x_{M}$, the relation $\mathbf{x}(M, N) \succ \mathbf{x}$ holds and for $x_{M}>x_{N}$, the ordering $\mathbf{x}(M, N) \succ \mathbf{x}$ is true. 
We will demonstrate the implication of patience in the profile for which $x_{N}>x_{M}$, the other case (namely, $x_{M}>x_{N}$ ) follows from a symmetric argument. Observe that (in either case) the definition of the profiles $\mathbf{x}(M, N)$ and $\mathbf{x}$ implies that for any $n \geq M+1$ we get

$$
\sum_{k=1}^{n} k\left(x_{k}(M, N)-x_{k}\right)=(M-N)\left(x_{N}-x_{M}\right)
$$

From (7) it follows that whenever $x_{N}>x_{M}$ we have $\sum_{k=1}^{n} k\left(x_{k}(M, N)-x_{k}\right)>0$ for all $n \geq M+1$, implying $\mathbf{x}(M, N) \succ \mathbf{x}$, showing that there is no impatience at $\mathbf{x}$. Clearly, the strict preference relation $(\mathbf{x}(M, N) \succ \mathbf{x})$ implies that $\succsim$ does not satisfy anonymity. Using the fact $\succsim$ is a subrelation to $\gtrsim$ we can now conclude that $\gtrsim$ is not anonymous and does not exhibit any impatience on $X$.

To show that $\gtrsim$ satisfies strong monotonicity, let $\mathbf{x}>\mathbf{y}$ and again as $\succsim$ is a subrelation to $\gtrsim$ it suffices to show that $\mathbf{x} \succ \mathbf{y}$. Clearly, $\mathbf{x}>\mathbf{y}$ implies that there is some $N$ such that $x_{N}>y_{N}$ and $x_{n} \geq y_{n}$ for all $n$. Hence, we must have for all $k \geq N$ that $\sum_{t=1}^{k} t\left(x_{t}-y_{t}\right)>0$ holds, implying $\mathbf{x} \succ \mathbf{y}$ from (5) as was needed.

\section{References}

[1] Banerjee K, Mitra T (2007) On the impatience implications of Paretian social welfare functions, Journal of Mathematical Economics 43: 236-248.

[2] Basu K, Mitra T (2003) Aggregating infinite utility streams under intergenerational equity, Econometrica 71: 1557-1563.

[3] Diamond P A (1965) The evaluation of infinite utility streams. Econometrica 33: 170-177.

[4] Lauwers L (2010) Ordering infinite utility streams comes at the cost of a non-Ramsey set. Journal of Mathematical Economics 46: 32-37.

[5] Lauwers L (2012) Intergenerational equity, efficiency, and constructibility. Economic Theory 49: 227-242.

[6] Mathias A R D (1977) Happy families. Annals of Pure and Applied Logic 12: 59-111.

[7] Ramsey FP (1928) On a problem of formal logic. Proceedings of the London Mathematical Society 2.30.4: 338-384.

[8] Svensson L G (1980) Equity among generations. Econometrica 48: 1251-1256.

[9] Szpilrajn E (1930) Sur l'extension de l'ordre partiel. Fundamenta Mathematicae 16: 386-389. 